\title{
Évolution de la surveillance de la qualité de l'air et prise en compte des enjeux sanitaires
}

\section{Evolution of the monitoring of the air quality and taking into account of the health stakes}

\section{Françoise FLESCH $^{*(1)}$, Alain TARGET ${ }^{(2)}$, Christine KOPFERSCHMITT ${ }^{(3)}$, Nadia IHADADENE ${ }^{(1)}$, Bausard LATRECH ${ }^{(1)}$}

(1) Centre Antipoison - Hôpitaux Universitaires de Strasbourg 5, rue de Madrid - 67309 SCHILTIGHEIM Cedex (3) Service de Pneumologie - Hôpitaux Universitaires de Strasbourg

* Auteur à qui adresser la correspondance : Docteur Françoise FLESCH, Centre Antipoison, 1, place de l'Hôpital BP 426 - 67091 STRASBOURG Cedex - Tél : 0388373737 - Fax : 0388115475

\section{RÉSUMÉ}

La pollution atmosphérique est l'un des grands problèmes actuels de santé publique. Dans la réglementation française, le décret 98360 du 6 mai 1998 et l'arrêté du 17 août 1998 ont défini les valeurs de référence pour les principaux indicateurs de pollution à savoir : le dioxyde d'azote (NO2), le dioxyde de soufre (SO2), les particules, l'ozone (O3), le plomb, le monoxyde de carbone $(\mathrm{CO})$, le benzène. Grâce, entre autres, à une politique nationale et locale d'évaluation et de gestion de la qualité de l'air ainsi qu'aux nombreuses évolutions technologiques, il a été noté une amélioration globale de la qualité de l'air. Néanmoins, ce bilan positif est terni par les problèmes que pose l'ozone à savoir : un accroissement inexorable des niveaux de fond et un dépassement fréquent des seuils de protection de la santé et de la végétation. D'autre part, des études épidémiologiques ont montré que les dangers sanitaires persistaient en dépit de cette amélioration globale de la qualité de l'air. Aussi est-il indispensable de poursuivre actions, efforts et études, afin de continuer à progresser dans la reconquête de la qualité de l'air.

\section{MOTS CLÉS}

Polluants atmosphériques, qualité de l'air, environnement.

\section{SUMMARY}

Air pollution is currently one of the major problems of public health. According to the French regulation, the reference values for the principal indicators of pollution have been defined by the decree 98360 of May 1998 and the ministerial order of August 1998 ; these are : nitrogen dioxide (NO2), sulphur dioxide (SO2), particles, ozone (O3), lead, carbon monoxide (CO), benzene. A global improvement of air quality has been noted thanks to, among other factors, national and local policies of air quality assessment and management together with a number of technological developments. Nevertheless, this positive global assessment is tarnished by ozone problems : the constant increase of basic ozone levels and the frequent encroaching of its limits set for the protection of people's health and of the vegetation. In addition, epidemiologic studies have demonstrated the persistence of health risks. Therefore it is important that actions, efforts and studies should continue in order to move on the way to the reconquest of good air quality.

\section{KEY-WORDS}

Air pollutants, air quality, environment. 


\section{Introduction}

La notion de pollution atmosphérique s'est imposée à partir des années 50 suite à une série d'épisodes majeurs de pollution, dont le smog de Londres en décembre 1952. En effet, alors qu'un épais brouillard avait envahi l'agglomération, il a été noté parmi la population, une recrudescence des troubles pulmonaires, digestifs et ORL ainsi qu'un excès de mortalité durant cette période. Les premières mesures des niveaux de pollution en France ont été réalisées à Paris vers la fin des années 50. Progressivement a été établie une corrélation entre des niveaux relativement faibles de polluants et des effets sanitaires en termes de mortalité et de morbidité. Aussi la France s'est-elle dotée le 30 décembre 1996 d'une nouvelle loi sur l'air qui s'est substituée aux textes de lois de 1948 et de 1961 devenus obsolètes. Les principales dispositions de cette loi ont été de définir les conditions de surveillance de la qualité de l'air et de ses effets, de fixer les modalités de mise en œuvre du droit à l'information et de prévoir un ensemble de mesures et d'outils de planification permettant de maîtriser ou d'améliorer la qualité de l'air.

Le dispositif français s'est fortement appuyé sur la politique de l'Union Européenne. Une directive cadre européenne est parue en 1996, sur l'évolution et la gestion de la qualité de l'air ambiant : elle concerne 13 polluants indicateurs qui sont : $\mathrm{SO}_{2}, \mathrm{NO}_{2}$, particules (PM10 et totales), $\mathrm{Pb}, \mathrm{O}_{3}, \mathrm{CO}$, benzène, hydrocarbures aromatiques polycycliques (HAP), cadmium, arsenic, nickel et mercure. Elle définit pour ces indicateurs des objectifs de qualité, des valeurs limites voir des seuils d'alerte pour le $\mathrm{SO}_{2}, \mathrm{NO}_{2}, \mathrm{O}_{3}$ et les particules ; elle précise également le champ d'intervention des réseaux et définit les méthodes de mesurage.

En France, il existe 39 réseaux de mesure ayant un statut d'association "loi 1901". Les stations sont équipées d'un ou plusieurs capteurs analyseurs, le plus souvent multi-paramétriques, fonctionnant de manière automatique continue, complétés de mesures mobiles et de modélisation. Le rôle de ces associations de surveillance est, non seulement de suivre l'évolution de la pollution, mais aussi, entre autres, de contribuer à la compréhension de ses mécanismes générateurs, de mieux prévoir les épisodes de pollution grâce à des platesformes de modélisations et de permettre la mise en place de mesures susceptibles de permettre l'amélioration de la qualité de l'air.

\section{Les principaux indicateurs de pollution}

Dans la réglementation française, le décret 98360 du 6 mai 1998 et l'arrêté du 17 août 1998 ont défini les valeurs de référence pour les principaux indicateurs de pollution à savoir : dioxyde d'azote $\left(\mathrm{NO}_{2}\right)$, dioxyde de soufre $\left(\mathrm{SO}_{2}\right)$, particules $(\mathrm{FN}=$ fumées noires), particules PM10 (particules fines de diamètre moyen $<10 \mu \mathrm{m})$, ozone $\left(\mathrm{O}_{2}\right)$, plomb, monoxyde de carbone (CO), benzène.

\section{LES OXYDES D'AZOTE (NOx)}

La famille des composés oxygénés de l'azote regroupe de nombreux composés chimiques $\left(\mathrm{NO}, \mathrm{NO}_{2}, \mathrm{~N}_{2} \mathrm{O}\right.$, $\mathrm{N}_{2} \mathrm{O}_{5} \ldots$ ). Néanmoins, dans le cadre de la réglementation, les $\mathrm{NOx}$ désignent l'ensemble $\mathrm{NO}+\mathrm{NO}_{2}$.

\section{Origine :}

Les NOx sont essentiellement produits lors des processus de combustion. Il s'agit de polluants générés à $50 \%$ par les transports au niveau national, ce pourcentage variant suivant la typologie (agglomération ou rural).

\section{Effets sur la santé :}

Le $\mathrm{NO}_{2}$ a des propriétés oxydantes et irritantes. Une exacerbation de la réaction bronchique a été noté chez certains patients asthmatiques pour des concentrations importantes.

\section{Effets sur l'environnement :}

Le $\mathrm{NO}_{2}$ augmente la sensibilité des végétaux aux agressions externes (déficit hydrique, maladies...).

Les NOx participent à l'acidification et à l'eutrophisation des milieux. Ils participent également à l'acidité des pluies et donc à la corrosion de certains matériaux type calcaire. Sous l'action du rayonnement solaire, ils contribuent à la formation d'ozone troposphérique.

\section{Principales mesures réglementaires et objectifs de la qualité de l'air :}

- objectif de qualité pour le $\mathrm{NO}_{2}$ :

$50 \mu \mathrm{g} / \mathrm{m}^{3}$ centile 50 des valeurs moyennes horaires prises sur toute l'année

$135 \mu \mathrm{g} / \mathrm{m}^{3}$ centile 95 des valeurs moyennes horaires prises sur toute l'année

- niveau de recommandation de la population : $200 \mu \mathrm{g} / \mathrm{m}^{3}$ sur 1 heure

- niveau d'alerte : $400 \mu \mathrm{g} / \mathrm{m}^{3}$ sur 1 heure

- mesures réglementaires :

1991 : mise en place de la taxe parafiscale sur les rejets de plus de 150 tonnes par an 
1993 : généralisation des pots catalytiques sur les voitures particulières à essence

1993/1998 : arrêté intégré prescrivant une norme de rejet à l'ensemble des installations industrielles.

1997 : pot catalytique sur les véhicules diesel.

\section{LE DIOXYDE DE SOUFRE $\left(\mathrm{SO}_{2}\right)$}

\section{Origine :}

Le $\mathrm{SO}_{2}$ provient principalement de procédés de combustion mettant en ouvre des combustibles fossiles soufrés (fiouls industriels et domestiques, gasoil ou charbon). Il s'agit d'un polluant essentiellement d'origine industrielle.

\section{Effets sur la santé :}

Le $\mathrm{SO}_{2}$ étant très soluble dans l'eau, il ne pénètre que très peu en profondeur dans l'arbre respiratoire. Il s'agit d'un gaz irritant pour lequel des études expérimentales ont montré qu'il induisait une plus grande sensibilité aux allergènes et favorisait la survenue de crises d'asthme mais pour des concentrations bien supérieures à celles rencontrées habituellement dans l'atmosphère.

\section{Effets sur l'environnement :}

Des nécroses végétales peuvent apparaître à partir de concentrations de $800 \mu \mathrm{g} / \mathrm{m}^{3}$ pour 8 heures d'exposition.

Le $\mathrm{SO}_{2}$ aggrave l'acidification des milieux et a un effet délétère sur le patrimoine bâti.

\section{Principales mesures réglementaires et objectifs de la qualité de l'air :}

- objectifs de qualité :

40 à $60 \mu \mathrm{g} / \mathrm{m}^{3}$ en moyenne annuelle

100 à $150 \mu \mathrm{g} / \mathrm{m}^{3}$ en moyenne journalière

- niveau de recommandation : $300 \mu \mathrm{g} / \mathrm{m}^{3}$ sur 1 heure

- niveau d'alerte : $600 \mu \mathrm{g} / \mathrm{m}^{3}$ sur 1 heure

- mesures réglementaires :

1991 : taxe parafiscale sur les rejets de plus de 150 tonnes/an

1994 puis 1996 : diminution de la teneur en soufre du gasoil et du fioul domestique.

\section{LES PARTICULES}

Selon leur taille, elles pénètrent plus ou moins profondément dans l'appareil respiratoire. D'un diamètre supérieur à $10 \mu \mathrm{m}$, elles sont principalement arrêtées au niveau des voies respiratoires. De 2,5 à $10 \mu \mathrm{m}$, elles atteignent l'arbre trachéo-bronchique. À moins de $2,5 \mu \mathrm{m}$, elles atteignent les alvéoles. L'action des particules est double ; elle est liée d'une part à la particule elle-même dont la composition est très variable selon l'origine, d'autre part aux polluants qu'elles transportent (hydrocarbures, métaux etc...).

\section{Origine :}

À l'échelle planétaire, une très grosse quantité de particules est émise par les océans, les volcans, et les feux de forêt. Toutefois, dans les pays industrialisés, les émissions d'origine anthropique sont importantes et proviennent principalement des transports, de l'industrie et du secteur résidentiel et tertiaire.

\section{Effets sur la santé :}

Les particules supérieures à $10 \mu \mathrm{m}$ (PM10) peuvent être à l'origine d'irritations respiratoire, cutanée et oculaire.

Les particules inférieures à $10 \mu \mathrm{m}$ (dont les plus préoccupantes actuellement sont les particules diesel dont la taille de 0,1 à $0,3 \mu \mathrm{m}$ leur permet de descendre jusqu'à l'alvéole) peuvent majorer les symptômes respiratoires chez des personnes sensibles (insuffisants respiratoires chroniques, asthmatiques...), et sont suspectées de jouer un rôle dans le développement de cancers du poumon. Des études expérimentales ont par ailleurs montré leur rôle adjuvant dans l'apparition de l'allergie.

\section{Effets sur l'environnement :}

Les particules sont à l'origine d'une baisse de croissance et de nécrose végétale notamment autour d'industries comme les cimenteries.

Elles modifient par ailleurs les propriétés superficielles des pierres et favorisent leur désagrégation.

Principales mesures réglementaires et objectifs de la qualité de l'air :

- objectifs de qualité :

$30 \mu \mathrm{g} / \mathrm{m}^{3}$ en moyenne annuelle des concentrations de particules inférieures ou égales à $10 \mu \mathrm{m}$ (PM 10)

40 à $60 \mu \mathrm{g} / \mathrm{m}^{3}$ en moyenne annuelle par la méthode des fumées noires

100 à $150 \mu \mathrm{g} / \mathrm{m}^{3}$ en moyenne journalière par la méthode des fumées noires

- principales dispositions réglementaires :

1997 : opacimétrie des poids lourds

1997 en Alsace : arrêté préfectoral (recommandation : $80 \mu \mathrm{g} / \mathrm{m}^{3} / 24 \mathrm{~h}$ et alerte $: 125 \mu \mathrm{g} / \mathrm{m}^{3} / 24 \mathrm{~h}$ ).

2000 : opacimétrie des voitures diesel.

\section{OZONE $\left(\mathrm{O}_{3}\right)$}

\section{Origine :}

Il s'agit d'un constituant normal de l'air présent dans la troposphère à très faible concentration. En cas d'épi- 
sodes de pollution, l'ozone est produit, suite à des réactions chimiques d'une grande complexité entre les divers polluants primaires émis par l'homme ou les sources naturelles dont les NOx et les composés organiques volatils. L'ensoleillement est propice à l'apparition de cette pollution photochimique et l'ozone seul est généralement mesuré en tant que traceur et indicateur de cette pollution.

\section{Effets sur la santé :}

Les altérations biologiques induites par l'ozone semblent principalement liées à ses propriétés oxydantes. Il s'agit d'un gaz peu soluble dans l'eau donc susceptible de pénétrer profondément dans les voies respiratoires. Il peut, selon la susceptibilité individuelle des sujets, provoquer des manifestations irritatives au niveau de la gorge, des yeux et des bronches avec les symptômes suivants : toux, gène thoracique, douleur à l'inspiration profonde, essoufflement, irritation nasale et pharyngée. Il diminue transitoirement les performances physiques chez le sportif et diminue le seuil de sensibilité aux allergènes chez les sujets asthmatiques.

\section{Effets sur l'environnement :}

Il existe une très grande variabilité de la sensibilité des plantes à l'ozone, les espèces les plus sensibles étant les herbacées, les pins, les mélèzes et le tabac.

L'ozone précipite le phénomène de vieillissement des plantes, perturbe la photosynthèse et réduit la durée du cycle végétatif.

Il participe par ailleurs à l'acidification des milieux et pourrait être impliqué dans la dégradation de divers matériaux comme les caoutchoucs, le papier, les encres.

\section{Objectifs de la qualité de l'air :}

- $65 \mu \mathrm{g} / \mathrm{m}^{3}$ en moyenne journalière pour la protection de la végétation

$110 \mu \mathrm{g} / \mathrm{m}^{3}$ en moyenne sur 8 heures pour la protection de la santé humaine.

$200 \mu \mathrm{g} / \mathrm{m}^{3}$ en moyenne horaire pour la protection de la végétation.

- niveau de recommandation de la population : $180 \mu \mathrm{g} / \mathrm{m}^{3}$ sur 1 heure.

- niveau d'alerte : $360 \mu \mathrm{g} / \mathrm{m}^{3}$ sur 1 heure.

\section{LE PLOMB}

\section{Origine :}

Étant donné la présence de plomb tétraéthyle dans les carburants, le trafic automobile était très largement responsable dans les émissions de plomb. Depuis l'apparition, début des années 90 , de l'essence sans plomb, l'air n'est plus une source majeure d'exposition.

\section{Effets sur la santé :}

L'intoxication chronique au plomb induit des symptômes principalement neurologiques en particulier des troubles du développement intellectuel et du comportement chez l'enfant. Le plomb dans l'air ne peut pas être responsable en soi de cette pathologie, mais peut contribuer à l'accumulation du plomb dans l'organisme.

\section{Principales mesures réglementaires et objectifs} de la qualité de l'air :

- objectif de qualité $0,5 \mu \mathrm{g} / \mathrm{m}^{3}$ en concentration moyenne annuelle.

- principales dispositions réglementaires :

1991 : arrêté ministériel relatif aux installations d'incinérations de déchets ménagers prescrivant une norme de rejet de $5 \mathrm{mg} / \mathrm{Nm}^{3}$ pour la somme de 6 métaux dont le plomb.

1996 : arrêté ministériel relatif aux installations d'incinération de déchets industriels spéciaux prescrivant une norme de rejet de $0,5 \mathrm{mg} / \mathrm{Nm}^{3}$ pour la somme de 12 métaux dont le plomb.

2000 : interdiction de l'essence plombée.

\section{LE MONOXYDE DE CARBONE}

\section{Origine :}

Le CO de l'air extérieur est un polluant essentiellement lié au transport et dont les émissions sont en régression depuis 1993, date d'introduction des pots catalytiques ; la concentration atmosphérique en $\mathrm{CO}$ mérite d'être surveillée dans les tunnels et les parkings souterrains mal ventilés.

\section{Effets sur la santé :}

Pour des niveaux d'exposition pouvant être mesurés en milieu urbain il a été noté la possibilité de troubles cardiaques, troubles de la vigilance, vertiges, céphalées, nausées.

\section{Effets sur l'environnement :}

L'effet du CO sur les végétaux peut aller d'une vacuolisation des cellules végétales à la mort de la plante.

\section{Objectifs de qualité :}

$10 \mathrm{mg} / \mathrm{m}^{3}$ en moyenne sur 8 heures.

\section{LE BENZÈNE}

\section{Origine :}

Le benzène est un polluant majoritairement émis par le trafic routier (perte par évaporation à partir de la châ̂ne de stockage des carburants, de la distribution, à par- 
tir du carburateur et du réservoir ; présence de benzène dans les gaz d'échappement du moteur).

\section{Effets sur la santé :}

À fortes doses, le benzène est cancérigène. Mais bien peu d'éléments permettent actuellement d'estimer le risque lié à la pollution benzénique atmosphérique.

\section{Objectifs de la qualité de l'air et principales mesures réglementaires :}

- objectif de qualité : $2 \mu \mathrm{g} / \mathrm{m}^{3}$ en moyenne annuelle

- la directive du 13 octobre 98 (dite auto-oil) relative à la qualité de l'essence et des carburants diesels fixe une nouvelle formulation des carburants; la teneur en ben-

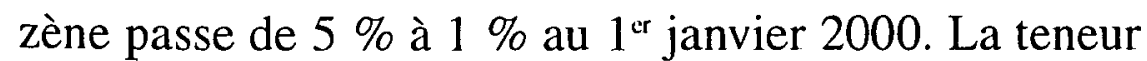
maximale en composés aromatiques passe de $50 \%$ à

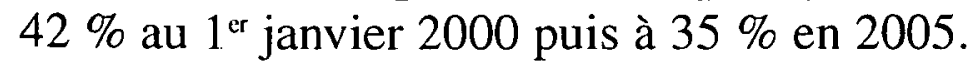

\section{Conclusion}

La pollution atmosphérique est l'un des grands problèmes actuels de santé publique. La surveillance de la qualité de l'air, les études épidémiologiques permettant d'appréhender progressivement la connaissance des effets de la qualité de l'air sur la santé, l'information de la population, les recommandations sanitaires, une politique nationale et locale d'évaluation et de gestion de la qualité de l'air, l'amélioration des contrôles, la sévérité des réglementations, les évolutions en matière de technologies des moteurs, les nouveaux carburants ... voici bon nombre d'éléments qui ont incontestablement conduit à une amélioration globale de la qualité de l'air. Néanmoins ce bilan positif souffre d'une exception majeure : l'ozone dont les niveaux de fond croissent inexorablement $(2,4 \%$ par an dans l'hémisphère nord) et dont les seuils relatifs à la protection de la santé et de la végétation sont souvent dépassés ce qui entraîne d'ailleurs une perte considérable du rendement des cultures agricoles (10 à $25 \%$ selon les cultures d'après des études américaines). D'autre part, en dépit de cette amélioration globale de la qualité de l'air des études épidémiologiques ont montré que les dangers sanitaires de la pollution atmosphérique persistaient. Aussi est-il indispensable de poursuivre actions, efforts et études afin de continuer à progresser dans la reconquête de la qualité de l'air et d'offrir ainsi, aux générations futures, la possibilité de respirer un air qui ne nuise pas à leur santé (article 1 de la loi sur l'air du 30 décembre 96).

\section{Références}

1. APPA. La pollution atmosphérique et l'homme : évaluer les expositions. Symposium international. Pollution atmosphérique : Dec 1999.

2. DRIRE Alsace. Plan régional pour la qualité de l'air en Alsace : Mai 2000.

3. Festy B., Dab W. Pollution atmosphérique. Encycl. Med. Chir. (Elsevier Paris) Toxicologie Pathologie Professionnelle, 1999 ; 16-001-C-10 : 12p.

4. Festy B. La pollution atmosphérique urbaine : sources, polluants et évolution. Bull. Acad. Nat. Med, $1997 ; 181$ $n^{\circ} 3: 461-76$.

5. Institut de veille sanitaire : surveillance épidémiologique air et santé. Surveillance des effets sur la santé liés à la pollution atmosphérique, Mars 1999.

6. Kuentz-Burchi Ch. Les polluants atmosphériques approche toxicologique de l'évaluation des risques. Thèse de médecine, Strasbourg 1996.

7. Pauli G., Kopferschmitt C., De Blay F. : Etudes expérimentales des effets des polluants atmosphériques. Allergie et Immunologie, $1998 ; 10: 317-322$

8. Pollution automobile et santé. Évolution des technologies, des carburants et de la réglementation. Actes du séminaire international 17-18 septembre 1999. 